\title{
Biological activities and volatile constituents of Daucus muricatus L. from Algeria
}

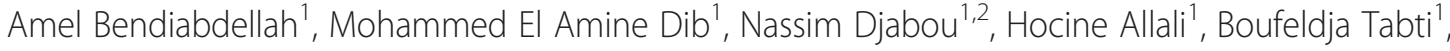
Alain Muselli $i^{*}$ and Jean Costa ${ }^{2}$

\begin{abstract}
Background: In order to find new bioactive natural products, the antimicrobial and antioxidant activities of essential oil components extracted from the separated organs of the Algerian medicinal and aromatic plant Daucus muricatus L. were studied.

Results: The chemical composition of essential oils obtained by hydrodistillation (HD) was investigated using Gas Chromatography-Retention Indices (GC-RI) and GC-Mass Spectrometry (GC-MS). Two types of essential oils were produced by $D$. muricatus: (i) The oil from roots is mainly composed by nonterpenic oxygenated compounds $(59.8 \mathrm{~g} / 100 \mathrm{~g}$ ), and (ii) the aerial part oils (i.e., the leaves, stems, flowers, and umbels) was mainly composed by terpenic hydrocarbon compounds $(62.3-72.2 \mathrm{~g} / 100 \mathrm{~g})$. The chemical composition of the volatile fraction isolated from different organs of Daucus muricatus were studied by HS-SPME/GC-RI and GC-MS after optimization of Solid Phase MicroExtraction parameters. For all organs studied, the main volatiles emitted by the plant were hydrocarbon compounds (60.7-82.2 g/100 g). Only quantitative differences between the volatiles of the separated organs studied were observed. In addition, the activity of the oil of D. muricatus against eight bacterial strains and one yeast was investigated. The oil from roots revealed active against $S$. aureus, while the essential oil obtained from the aerial parts was active against the yeast $C$. albicans.
\end{abstract}

Conclusions: Daucus muricatus essential oil seems be a promising source of natural products with potential antimicrobial activity.

Keywords: Daucus muricatus. L, Essential oils, HS-SPME, GC/MS, Antimicrobial and antioxidant activities

\section{Background}

Daucus is a genus belonging to the Apiaceae family and consists of about 600 species that are widely distributed around the world. In Algeria, the Daucus genus is represented by more than 27 species living in dry and uncultivated areas [1], and they are mostly found from Tlemcen to Mascara [1,2]. The most prevalent of the species is Daucus carota L. (carrot) reported with eight subspecies throughout Algeria [1]. Daucus muricatus L., synonym of Artedia muricata L., Caucalis muricata L., and Platyspermum muricatum Hoffm., is widely distributed in Algeria, Spain, Portugal, Corsica, Sardinia, Sicily, Italy, the Aegean Islands, and Turkey [2]. Daucus muricatus is an annual plant $30-50 \mathrm{~cm}$ high, dark green,

\footnotetext{
* Correspondence: muselli@univ-corse.fr

2 UMR CNRS 6134, Laboratoire Chimie des Produits Naturels, Campus

Grimaldi, Université de Corse, BP 52, 20250, Corte, France

Full list of author information is available at the end of the article
}

bristling at the base, with a stem thickened at the nodes and branches spreading erect. The leaves are soft and lanceolate in their periphery in segments cut into narrow strips with white flowers. The umbels opposite the leaves at the end are contracted, the fruit are large, elliptical and compressed, armed with spines expanded and confluent at the base [1,2]. Several investigations deal with the chemical composition of essential oils of the Daucus species [3-27]. While no study has investigated D. muricatus essential oils, most of them have reported the chemical composition of essential oils from $D$. carota and its subspecies [3,4,6-16,20,22,23,25-27]. However, only three studies have reported the chemical composition of essential oils from Daucus species from Algeria. The first reported the chemical composition of the essential oil of D. reboudii Coss. [17], and the other two reported the chemical composition of the oil from D. crinitus Desf. $[18,19]$. Previous reports showed that

\section{(C) Chemistry Central}


the chemical composition of Daucus species was more dominated by monoterpene hydrocarbon compounds such $\alpha$-pinene and sabinene $[3,4,10,14,15]$, and occasionally by phenylpropanoids compounds such as apiol, myristicin, and isochavicol [3,14-16]. Several studies recently investigated the biological activity of Daucus essential oils $[6,10,12,19,20]$. However, there remain many species and subspecies of Daucus that have not yet been examined.

As part of our ongoing chemical investigation of the essential oils from the Algerian Daucus genus [18] and our search for active natural products to fight nosocomial infections, we investigated for the first time the chemical composition and biological activities of Daucus muricatus L. through the study of: (i) the volatile components of $D$. muricatus roots, leaves, stems, flowers, and umbels extracted by hydrodistillation and by solid phase microextraction (SPME) using gas chromatography (GC) and gas chromatography-mass spectrometry (GC-MS), (ii) the antibacterial activity of D. muricatus essential oil against nine species of microorganisms involved in nosocomial infections using paper disc diffusion and agar dilution methods.

\section{Results and discussion}

\section{Essential oil chemical composition}

GC-RI and GC-MS analysis of D. muricatus essential oils obtained from the roots, stems, leaves, flowers, and umbels that accounted for 92.8, 94.7, 94.5, 95.4, and $95.7 \mathrm{~g} / 100 \mathrm{~g}$ of the oils, respectively and allowed the identification of 99 compounds. Their retention indices, yields, and relative concentrations expressed in $\mathrm{g} / 100 \mathrm{~g}$ of essential oil are shown in Table 1. Among these, 39 monoterpenes, 32 sesquiterpenes, 22 nonterpenic compounds, three diterpenes, two phenylpropanoids, and one $\mathrm{C}_{13}$-isopropenoid were identified. All components were identified by comparison of their EI-MS and GCretention indices with those of our laboratory-produced "Arômes" library, with the exception of nine components that were identified by comparison with spectral data and retention indices from the literature (see Table 1). Two types of essential oils were produced by D. muricatus. The root oils are mainly composed by oxygenated compounds (59.8 g/100 g), and the aerial part oils (i.e., the leaves, stems, flowers, and umbels) were dominated by the occurrence of hydrocarbon compounds $(62.3-72.2 \mathrm{~g} / 100 \mathrm{~g})$. The main components of root oil were nonterpenic aliphatic compounds that accounted for $56.7 \mathrm{~g} / 100 \mathrm{~g}$, such as eicosane (18.6 g/ $100 \mathrm{~g})$, undecan-2-one (10.2 g/100 g), and tridecanol $(6.4 \mathrm{~g} / 100 \mathrm{~g})$. Conversely, the main components of aerial organs of D. muricatus were monoterpene hydrocarbons $(52.0-58.5 \mathrm{~g} / 100 \mathrm{~g})$. For all organs studied, limonene $(21.9-24.0 \mathrm{~g} / 100 \mathrm{~g})$ and $\alpha$-pinene $(9.9-21.8 \mathrm{~g} / 100 \mathrm{~g})$ were the main components. Their relative abundances were followed by that of sabinene $(4.7-8.1 \mathrm{~g} / 100 \mathrm{~g})$ in stems, leaves and flowers, and trans-sabinyl acetate in umbels $(12.1 \mathrm{~g} / 100 \mathrm{~g})$. On moving from the bottom to the top of the plant, we noted that the relative concentrations of nonterpenic compounds decreased as follows: $56.7 \mathrm{~g} / 100 \mathrm{~g}$ in the roots, $12.5 \mathrm{~g} / 100 \mathrm{~g}$ in the stems, $7.7 \mathrm{~g} / 100 \mathrm{~g}$ in the leaves, $4.0 \mathrm{~g} / 100 \mathrm{~g}$ in the flowers, and then $3.6 \mathrm{~g} / 100 \mathrm{~g}$ in the umbels.

\section{HS-SPME analysis}

The volatiles emitted from the D. muricatus roots, leaves, stems, umbels, and flowers were investigated using HS-SPME under optimized parameters. The optimization of the HS-SPME sampling parameters was conducted using fresh plant material based on the sum of the total peak areas obtained using GC-FID. The maximum sum of the total peak area was acquired for an equilibrium and extraction temperature of $70^{\circ} \mathrm{C}$, an equilibrium time of $60 \mathrm{~min}$, and an extraction time of $30 \mathrm{~min}$. The GC-RI and GC-MS analysis identified 78 components: 42 monoterpenes, 18 nonterpenic compounds, 16 sesquiterpenes, and two phenylpropanoids (Table 2). Identification of 74 components was conducted by comparing their EI-MS and retention indices with those in our laboratory-produced "Arômes" library, and four components were identified by comparing their EI-MS data and their apolar retention indices with those reported in the literature and in commercial libraries. Regarding the organ contribution to the aromatic plant fingerprint, it should be noted that the volatile constituents were more abundant in the leaves than in the other parts of the plant. Our analysis showed that the chemical composition of the HS fractions obtained from different organs was qualitatively similar but differed by the relative amounts of the main components. Relative to $D$. muricatus oil, the main volatiles emitted by plant were hydrocarbon compounds (60.7-82.2\%) for all organs studied. More precisely, the sum of hydrocarbon monoterpenes $(33.6 \%-64.1 \%)$ and hydrocarbon nonterpenic compounds $(6.4 \%-25.5 \%)$ was higher than that of oxygenated compounds, which never accounted for more than $23.9 \mathrm{~g} / 100 \mathrm{~g}$. The main volatile components of roots were terpinolene (10.2\%), bornyl acetate $(9.7 \%)$, p-cymene (9.1\%), $\alpha$-pinene (8.7\%), and undecane (7.2\%). The relative amounts of terpinolene $(0.4 \%-1.3 \%)$ and $\mathrm{p}$-cymene $(3.1 \%-3.4 \%)$ were lower in the aerial organs, and the main volatile emitted by leaves, flowers and umbels was limonene $(30.6 \%, 19.1 \%$, and $28.1 \%$, respectively). In the stems, limonene (13.3\%) was present in lower amounts than undecane (16.9\%), which was identified as a major component. In addition, undecane was produced in appreciable amounts in leaves, flowers, and umbels $(6.1 \%, 3.6 \%$, and $7.4 \%$, respectively). They were 
Table 1 Composition of the essential oils of $D$. muricatus (roots, leaves, stems, flowers and umbels)

\begin{tabular}{|c|c|c|c|c|c|c|c|c|c|c|c|}
\hline \multirow[b]{2}{*}{$\mathrm{N}^{\circ}$} & \multirow[b]{2}{*}{ Components $^{a}$} & \multirow[b]{2}{*}{$\mathrm{IRI}^{\mathrm{b}}$} & \multirow[b]{2}{*}{$\mathrm{Rla}^{\mathrm{C}}$} & \multirow[b]{2}{*}{ Rlp $^{d}$} & \multirow[b]{2}{*}{ Aerial parts } & \multicolumn{5}{|c|}{ Separated organs ${ }^{e}$} & \multirow[b]{2}{*}{ Identification ${ }^{f}$} \\
\hline & & & & & & Stems & Leaves & Flowers & Umbels & Roots & \\
\hline 1 & Nonane & 900 & 898 & 899 & 1.0 & 0.5 & 1.3 & 1.1 & 0.3 & - & $\mathrm{RI}, \mathrm{MS}$ \\
\hline 2 & a-Thujene & 932 & 923 & 1011 & 0.6 & $\operatorname{tr}$ & 0.2 & 0.7 & 0.1 & - & $\mathrm{Rl}, \mathrm{MS}$ \\
\hline 3 & a- Pinene & 936 & 931 & 1016 & 16.7 & 9.9 & 18.9 & 14.3 & 21.8 & 0.5 & $\mathrm{Rl}, \mathrm{MS}$ \\
\hline 4 & Camphene & 950 & 944 & 1056 & 0.3 & 0.2 & 0.2 & 0.2 & 0.8 & $\operatorname{tr}$ & $\mathrm{RI}, \mathrm{MS}$ \\
\hline 5 & Thuja-2,4 (10) diene & 946 & 945 & 1085 & $\operatorname{tr}$ & 0.2 & 0.1 & $\operatorname{tr}$ & - & - & $\mathrm{Rl}, \mathrm{MS}$ \\
\hline 6 & Sabinene & 973 & 967 & 1111 & 18.9 & 5.1 & 4.7 & 8.1 & 4.6 & 0.2 & $\mathrm{Rl}, \mathrm{MS}$ \\
\hline 7 & $\beta$-pinene & 978 & 970 & 1102 & 2.5 & 2.1 & 1.8 & 2.8 & 1.4 & 0.1 & $\mathrm{RI}, \mathrm{MS}$ \\
\hline 8 & Myrcene & 987 & 980 & 1152 & 1.8 & 2.1 & 2.3 & 1.6 & 2.6 & - & $\mathrm{Rl}, \mathrm{MS}$ \\
\hline 9 & a-Phellandrene & 1002 & 997 & 1140 & 0.6 & 0.5 & 0.4 & 0.6 & 0.2 & - & $\mathrm{Rl}, \mathrm{MS}$ \\
\hline 10 & a-Terpinene & 1013 & 1008 & 1158 & 1.1 & 1.1 & 1.1 & 1.5 & 0.8 & 0.1 & $\mathrm{RI}, \mathrm{MS}$ \\
\hline 11 & p-Cymene & 1015 & 1012 & 1147 & 1.3 & 4.4 & 1.4 & 0.9 & 1.4 & 0.1 & $\mathrm{Rl}, \mathrm{MS}$ \\
\hline 12 & Limonene & 1025 & 1022 & 1195 & 14.2 & 22.6 & 21.3 & 24.0 & 21.9 & 0.3 & $\mathrm{RI}, \mathrm{MS}$ \\
\hline 13 & (Z)- $\beta$-Ocimene & 1029 & 1025 & 1215 & 0.1 & 0.1 & 0.1 & $\operatorname{tr}$ & 0.2 & $\mathrm{tr}$ & $\mathrm{RI}, \mathrm{MS}$ \\
\hline 14 & Y-Terpinene & 1051 & 1049 & 1239 & 2.6 & 2.4 & 1.6 & 3.1 & 1.1 & $\operatorname{tr}$ & $\mathrm{Rl}, \mathrm{MS}$ \\
\hline 15 & trans-Sabinene hydrate & 1053 & 1052 & 1438 & 0.2 & 0.1 & 0.1 & 0.3 & $\operatorname{tr}$ & - & $\mathrm{Rl}, \mathrm{MS}$ \\
\hline 16 & Nonan-2-one & 1070 & 1073 & 1392 & 0.1 & 0.6 & 0.1 & $\operatorname{tr}$ & 0.1 & 0.3 & $\mathrm{Rl}, \mathrm{MS}$ \\
\hline 17 & p-Cymenene & 1075 & 1077 & 1420 & 0.6 & 0.7 & 0.1 & $\operatorname{tr}$ & $\operatorname{tr}$ & 0.1 & $\mathrm{Rl}, \mathrm{MS}$ \\
\hline 18 & Terpinolene & 1082 & 1079 & 1292 & 0.3 & 0.6 & 0.3 & 0.7 & 0.2 & 0.1 & $\mathrm{Rl}, \mathrm{MS}$ \\
\hline 19 & Linalool & 1083 & 1085 & 1392 & 0.1 & $\operatorname{tr}$ & 0.4 & 0.3 & 0.2 & - & $\mathrm{Rl}, \mathrm{MS}$ \\
\hline 20 & Undecane & 1100 & 1100 & 1098 & 0.6 & 2.1 & 1.9 & 0.4 & 0.9 & 0.1 & $\mathrm{Rl}, \mathrm{MS}$ \\
\hline 21 & Limonene-1,2-epoxide & 1117 & 1119 & 1446 & 0.2 & 0.2 & 0.2 & 0.3 & 0.4 & 0.9 & $\mathrm{Rl}, \mathrm{MS}$ \\
\hline 22 & trans-Pinocarveol & 1126 & 1120 & 1632 & 0.3 & 0.7 & 0.1 & 0.2 & 0.2 & 0.2 & $\mathrm{RI}, \mathrm{MS}$ \\
\hline 23 & trans-2-Nonenal & 1135 & 1133 & 1525 & 0.2 & 1.2 & 0.4 & 0.1 & 0.2 & 1.1 & $\mathrm{Rl}, \mathrm{MS}$ \\
\hline 24 & Pinocarvone & 1137 & 1135 & 1520 & 0.1 & 0.4 & 0.1 & 0.1 & tr & 0.1 & $\mathrm{Rl}, \mathrm{MS}$ \\
\hline 25 & Borneol & 1150 & 1149 & 1670 & 0.1 & 0.2 & 0.1 & $\operatorname{tr}$ & 0.1 & $\mathrm{tr}$ & $\mathrm{Rl}, \mathrm{MS}$ \\
\hline 26 & Cryptone & 1160 & 1159 & 1642 & 0.2 & 0.5 & 0.3 & 0.1 & 0.3 & 0.2 & $\mathrm{Rl}, \mathrm{MS}$ \\
\hline 27 & Terpinen-4-ol & 1164 & 1160 & 1563 & 2.7 & 1.1 & 0.8 & 3.1 & 0.8 & 1.7 & $\mathrm{RI}, \mathrm{MS}$ \\
\hline 28 & Decan-2-one & 1176 & 1170 & 1503 & 0.1 & 0.8 & 0.3 & 0.2 & 0.1 & 0.2 & $\mathrm{Rl}, \mathrm{MS}$ \\
\hline 29 & a-Terpineol & 1176 & 1177 & 1685 & 0.2 & 0.3 & 0.1 & 0.1 & tr & $\operatorname{tr}$ & $\mathrm{Rl}, \mathrm{MS}$ \\
\hline 30 & Myrtenol & 1178 & 1182 & 1763 & $\operatorname{tr}$ & 0.5 & 0.1 & 0.1 & 0.2 & 0.7 & $\mathrm{Rl}, \mathrm{MS}$ \\
\hline 31 & Decanal & 1188 & 1185 & 1481 & $\operatorname{tr}$ & 0.8 & 0.1 & 0.1 & tr & 0.2 & $\mathrm{Rl}, \mathrm{MS}$ \\
\hline 32 & Dodecane & 1200 & 1199 & 1201 & $\operatorname{tr}$ & 0.2 & 0.1 & $\operatorname{tr}$ & 0.1 & 0.3 & $\mathrm{Rl}, \mathrm{MS}$ \\
\hline 33 & Citronellol & 1213 & 1211 & 1724 & 0.1 & 0.1 & 0.1 & $\operatorname{tr}$ & - & $\operatorname{tr}$ & $\mathrm{Rl}, \mathrm{MS}$ \\
\hline 34 & Carvone & 1214 & 1215 & 1749 & $\operatorname{tr}$ & 0.2 & 0.1 & $\operatorname{tr}$ & - & $\operatorname{tr}$ & $\mathrm{Rl}, \mathrm{MS}$ \\
\hline 35 & Pulegone & 1215 & 1216 & 1602 & $\operatorname{tr}$ & 0.1 & $\operatorname{tr}$ & $\operatorname{tr}$ & - & - & $\mathrm{Rl}, \mathrm{MS}$ \\
\hline 36 & p-Anisaldehyde & 1218 & 1219 & 2049 & $\operatorname{tr}$ & 0.6 & $\operatorname{tr}$ & $\operatorname{tr}$ & 0.3 & - & $\mathrm{Rl}, \mathrm{MS}$ \\
\hline 37 & Geraniol & 1235 & 1235 & 1799 & 0.1 & 0.1 & 0.1 & 0.2 & - & $\operatorname{tr}$ & $\mathrm{Rl}, \mathrm{MS}$ \\
\hline 38 & trans-Myrtanol & 1240 & 1236 & 1858 & $\operatorname{tr}$ & 0.1 & 0.2 & 0.2 & 0.5 & - & $\mathrm{Rl}, \mathrm{MS}$ \\
\hline 39 & cis-Chrysanthenyl acetate & 1253 & 1242 & 1548 & 0.3 & 2.2 & 0.5 & $\operatorname{tr}$ & 0.1 & 0.2 & $\mathrm{RI}, \mathrm{MS}$ \\
\hline 40 & a-Terpinen-7-al & 1257 & 1256 & 1763 & 0.1 & 0.2 & 0.2 & 0.4 & 0.3 & $\operatorname{tr}$ & $\mathrm{Rl}, \mathrm{MS}$ \\
\hline 41 & Thymol & 1267 & 1264 & 2149 & $\operatorname{tr}$ & 0.1 & 0.1 & 0.1 & - & 0.6 & $\mathrm{Rl}, \mathrm{MS}$ \\
\hline 42 & Bornyl acetate & 1270 & 1266 & 1536 & 0.2 & 0.6 & 0.2 & 0.1 & 0.4 & 0.9 & $\mathrm{Rl}, \mathrm{MS}$ \\
\hline 43 & Undecan-2-one & 1273 & 1270 & 1579 & 0.3 & 3.9 & 0.5 & 0.3 & 0.8 & 10.2 & $\mathrm{Rl}, \mathrm{MS}$ \\
\hline 44 & trans-Sabinyl acetate & 1278 & 1271 & 1650 & 2.6 & 1.5 & 0.7 & 0.2 & 12.1 & 3.1 & $\mathrm{RI}, \mathrm{MS}$, Ref1 \\
\hline 45 & Carvacrol & 1278 & 1278 & 2224 & $\operatorname{tr}$ & 0.2 & 0.4 & $\operatorname{tr}$ & 0.1 & 0.5 & $\mathrm{Rl}, \mathrm{MS}$ \\
\hline 46 & Undecan-2-ol & 1287 & 1285 & 1723 & 0.1 & 0.3 & 0.1 & $\operatorname{tr}$ & - & 1.2 & $\mathrm{RI}, \mathrm{MS}$ \\
\hline
\end{tabular}


Table 1 Composition of the essential oils of $\boldsymbol{D}$. muricatus (roots, leaves, stems, flowers and umbels) (Continued)

\begin{tabular}{|c|c|c|c|c|c|c|c|c|c|c|c|}
\hline$\overline{47}$ & Myrtenyl acetate & 1332 & 1320 & 1701 & 0.1 & 0.1 & 0.2 & 0.1 & tr & 0.3 & $\mathrm{RI}, \mathrm{MS}$ \\
\hline 48 & 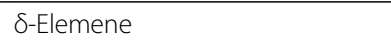 & 1340 & 1337 & 1535 & 0.2 & 0.5 & 1.7 & 0.1 & 0.2 & - & $\mathrm{Rl}, \mathrm{MS}$ \\
\hline 49 & Geranyl acetate & 1362 & 1360 & 1715 & $\mathrm{tr}$ & 0.2 & $\mathrm{tr}$ & 2.3 & - & 0.2 & $\mathrm{RI}, \mathrm{MS}$ \\
\hline 50 & Undecanol & 1363 & 1365 & 1820 & 0.2 & 0.6 & 1.1 & 1.2 & 0.1 & 0.3 & $\mathrm{Rl}, \mathrm{MS}$ \\
\hline 51 & a-Copaene & 1379 & 1377 & 1488 & 0.2 & 0.6 & 0.5 & 0.2 & 0.5 & - & $\mathrm{Rl}, \mathrm{MS}$ \\
\hline 52 & $\beta$-Bourbonene & 1379 & 1383 & 1496 & $\operatorname{tr}$ & 0.4 & 0.3 & 0.2 & 0.1 & - & $\mathrm{Rl}, \mathrm{MS}$ \\
\hline 53 & $\beta$-Elemene & 1389 & 1390 & 1570 & 0.3 & 0.3 & 0.1 & 0.2 & 0.5 & - & $\mathrm{Rl}, \mathrm{MS}$ \\
\hline 54 & Dodecanal & 1389 & 1395 & 1673 & $\operatorname{tr}$ & 0.1 & 0.1 & 0.2 & tr & 1.9 & $\mathrm{RI}, \mathrm{MS}$ \\
\hline 55 & Aristolene & 1418 & 1420 & 1553 & 0.1 & $\operatorname{tr}$ & 0.1 & 0.3 & 0.1 & & $\mathrm{Rl}, \mathrm{MS}$ \\
\hline 56 & trans-Caryophyllene & 1424 & 1422 & 1586 & 1.8 & 0.6 & 3.8 & 2.4 & 2.1 & 0.3 & $\mathrm{RI}, \mathrm{MS}$ \\
\hline 57 & Geranyl acetone & 1429 & 1426 & 1842 & 0.1 & 0.4 & 0.2 & 0.3 & 0.4 & 0.1 & $\mathrm{Rl}, \mathrm{MS}$ \\
\hline 58 & $\beta$-Copaene & 1430 & 1430 & 1579 & $\operatorname{tr}$ & 0.1 & 0.1 & $\operatorname{tr}$ & 0.4 & - & $\mathrm{Rl}, \mathrm{MS}$ \\
\hline 59 & a-Humulene & 1455 & 1450 & 1655 & 0.4 & 0.4 & 0.5 & 0.3 & 0.4 & 0.5 & $\mathrm{RI}, \mathrm{MS}$ \\
\hline 60 & $\beta$-lonone & 1468 & 1460 & 1902 & 0.5 & 0.2 & 0.1 & 0.8 & 0.4 & - & $\mathrm{Rl}, \mathrm{MS}$ \\
\hline 61 & Dodecanol & 1472 & 1468 & 1754 & 0.2 & 0.2 & 0.2 & $\mathrm{tr}$ & 0.1 & 3.7 & $\mathrm{RI}, \mathrm{MS}$ \\
\hline 62 & Y-Muurolene & 1473 & 1471 & 1667 & 0.1 & 0.9 & 0.3 & 0.1 & - & - & $\mathrm{Rl}, \mathrm{MS}$ \\
\hline 63 & Germacrene D & 1479 & 1476 & 1665 & 1.5 & 0.2 & 1.6 & 1.4 & 2.9 & 1.5 & $\mathrm{Rl}, \mathrm{MS}$ \\
\hline 64 & trans- $\beta$-Bergamotene & 1480 & 1475 & 1598 & $\operatorname{tr}$ & $\operatorname{tr}$ & $\operatorname{tr}$ & 0.1 & $\operatorname{tr}$ & 0.8 & $\mathrm{RI}, \mathrm{MS}$ \\
\hline$\overline{65}$ & 6-epi-Shyobunone & 1481 & 1480 & 1855 & 0.1 & 0.1 & 0.1 & 0.2 & 0.3 & 0.3 & $\mathrm{RI}, \mathrm{MS}$ \\
\hline 66 & $\gamma$-Humulene & 1483 & 1487 & 1682 & 0.2 & 0.4 & 0.2 & 0.4 & 0.1 & - & $\mathrm{Rl}, \mathrm{MS}$ \\
\hline 67 & Bicyclogermacrene & 1494 & 1490 & 1706 & 0.4 & 0.4 & 0.3 & 0.2 & 0.2 & - & $\mathrm{Rl}, \mathrm{MS}$ \\
\hline 68 & a-Muurolene & 1496 & 1498 & 1710 & 0.4 & 0.2 & 0.3 & 0.4 & 0.2 & - & $\mathrm{Rl}, \mathrm{MS}$ \\
\hline 69 & Shyobunone & 1500 & 1501 & 1897 & 0.1 & 0.5 & 0.5 & 0.4 & 0.2 & 0.1 & $\mathrm{RI}, \mathrm{MS}$ \\
\hline 70 & S-Cadinene & 1520 & 1515 & 1715 & 0.8 & 0.2 & 0.6 & 0.6 & 1.7 & 0.5 & $\mathrm{RI}, \mathrm{MS}$ \\
\hline 71 & E-a-Bisabolene & 1531 & 1526 & 1733 & 0.6 & 0.8 & 1.6 & 0.4 & 1.2 & 0.2 & $\mathrm{Rl}, \mathrm{MS}$ \\
\hline 72 & Isochavicol isobutyrate & 1541 & 1538 & 2136 & 5.3 & 1.2 & 2.2 & 6.7 & 1.6 & 2.3 & $\mathrm{Rl}, \mathrm{MS}$ \\
\hline 73 & Germacrene B & 1552 & 1555 & 1794 & 0.2 & 0.6 & 0.2 & 0.2 & 0.8 & $\operatorname{tr}$ & RI, MS, Ref1 \\
\hline 74 & 1,5-Epoxy-salvial-4(14)-ene & 1561 & 1561 & 1903 & 0.1 & 0.7 & 0.4 & 0.5 & 0.4 & 3.6 & $\mathrm{RI}, \mathrm{MS}$ \\
\hline 75 & Spathulenol & 1572 & 1564 & 2091 & 0.5 & 1.2 & 1.4 & 0.3 & 0.6 & - & $\mathrm{Rl}, \mathrm{MS}$ \\
\hline 76 & Caryophyllene oxide & 1578 & 1582 & 1943 & tr & 0.1 & 0.3 & 0.1 & $\operatorname{tr}$ & 0.5 & $\mathrm{Rl}, \mathrm{MS}$ \\
\hline 77 & Tridecanol & 1580 & 1586 & 2034 & - & $\operatorname{tr}$ & $\operatorname{tr}$ & $\operatorname{tr}$ & $\operatorname{tr}$ & 6.4 & RI, MS, Ref2 \\
\hline 78 & Viridiflorol & 1590 & 1586 & 2071 & 0.1 & 0.9 & 0.3 & 0.5 & 0.2 & & $\mathrm{RI}, \mathrm{MS}$ \\
\hline 79 & Copaborneol & 1592 & 1595 & 2142 & 0.5 & 0.8 & 0.3 & 1.5 & 0.1 & 1.4 & $\mathrm{RI}, \mathrm{MS}$ \\
\hline 80 & Guaia-6,10(14)-diene-4ß-ol & 1610 & 1609 & 2119 & 1.1 & 2.5 & 2.1 & 6.6 & 0.5 & 0.9 & RI, MS, Ref1 \\
\hline 81 & epi-Cubenol & 1621 & 1623 & 2046 & 0.2 & 0.3 & 0.2 & 0.4 & 0.3 & 0.9 & $\mathrm{Rl}, \mathrm{MS}$ \\
\hline 82 & Cubenol & 1630 & 1631 & 2001 & 0.5 & 0.2 & 0.2 & 0.3 & $\operatorname{tr}$ & $\operatorname{tr}$ & $\mathrm{Rl}, \mathrm{MS}$ \\
\hline 83 & T-Muurolol & 1633 & 1635 & 2156 & 0.5 & 0.7 & 0.3 & 0.6 & 0.7 & 1.4 & $\mathrm{Rl}, \mathrm{MS}$ \\
\hline 84 & a-Cadinol & 1643 & 1644 & 2212 & 0.5 & 0.7 & 0.5 & 0.6 & 0.3 & 0.9 & $\mathrm{RI}, \mathrm{MS}$ \\
\hline 85 & Isochavicol 2-methyl butyrate & 1651 & 1654 & 2256 & 0.1 & 0.3 & 0.2 & 0.2 & - & 0.4 & $\mathrm{RI}, \mathrm{MS}$ \\
\hline 86 & (Z)-a-Santalol & 1669 & 1665 & 2306 & 0.1 & 0.4 & 0.1 & 0.1 & 0.1 & - & $\mathrm{RI}, \mathrm{MS}$ \\
\hline 87 & Eudesma-4(15),7-dien-1 $\beta$-ol & 1671 & 1672 & 2346 & 0.1 & 0.3 & 0.2 & 0.1 & 0.5 & 1.3 & $\mathrm{Rl}, \mathrm{MS}$ \\
\hline 88 & (E,Z)-Farnesol & 1685 & 1680 & 2313 & $\operatorname{tr}$ & 0.2 & 0.1 & 0.1 & 0.1 & 3.1 & $\mathrm{Rl}, \mathrm{MS}$ \\
\hline 89 & Heptadecane & 1700 & 1699 & 1698 & $\operatorname{tr}$ & 0.3 & 0.3 & 0.1 & 0.1 & 0.5 & $\mathrm{RI}, \mathrm{MS}$ \\
\hline 90 & Tetradecanoic acid & 1761 & 1756 & 2651 & $\operatorname{tr}$ & 0.1 & 0.2 & 0.2 & - & 1.7 & RI, MS, Ref2 \\
\hline 91 & Neophytadiene & 1807 & 1806 & 1920 & 0.1 & 0.2 & 1.7 & 0.1 & 0.1 & 1.3 & RI, MS, Ref1 \\
\hline 92 & Diisobutyl ester & 1826 & 1826 & 2525 & 0.2 & 0.1 & 0.4 & $\operatorname{tr}$ & $\operatorname{tr}$ & 0.9 & RI, MS, Ref2 \\
\hline 93 & 6,10,14-Trimethylpentadecanone & 1845 & 1842 & 2125 & 0.2 & 0.1 & 0.4 & $\operatorname{tr}$ & 0.1 & 1.8 & $\mathrm{Rl}, \mathrm{MS}$, Ref2 \\
\hline 94 & Hexadecanoic acid & 1951 & 1956 & 2821 & 0.1 & 0.2 & $\operatorname{tr}$ & $\operatorname{tr}$ & 0.2 & 3.1 & RI, MS, Ref1 \\
\hline
\end{tabular}


Table 1 Composition of the essential oils of $\boldsymbol{D}$. muricatus (roots, leaves, stems, flowers and umbels) (Continued)

\begin{tabular}{|c|c|c|c|c|c|c|c|c|c|c|c|}
\hline 95 & Eicosane & 2000 & 2000 & 1998 & $\operatorname{tr}$ & 0.1 & $\operatorname{tr}$ & $\operatorname{tr}$ & 0.2 & 18.6 & $\mathrm{Rl}, \mathrm{MS}$ \\
\hline 96 & (Z)-Phytol & 2080 & 2085 & 2611 & 0.2 & 0.2 & 0.8 & 0.1 & 0.3 & - & $\mathrm{Rl}, \mathrm{MS}$ \\
\hline 97 & (E)-Phytol & 2114 & 2119 & 2568 & 0.3 & 0.2 & 1.9 & $\mathrm{tr}$ & 0.1 & - & $\mathrm{Rl}, \mathrm{MS}$ \\
\hline 98 & Tricosane & 2300 & 2302 & 2299 & 0.1 & 0.2 & $\operatorname{tr}$ & $\operatorname{tr}$ & 0.1 & 1.5 & $\mathrm{Rl}, \mathrm{MS}$ \\
\hline \multirow[t]{11}{*}{99} & Pentacosane & 2500 & 2498 & 2501 & 0.1 & 0.1 & 0.2 & 0.1 & 0,2 & 2.7 & $\mathrm{Rl}, \mathrm{MS}$ \\
\hline & Total identification $\mathrm{g} / 100 \mathrm{~g}$ & & & & 90.9 & 93.7 & 93.6 & 98.7 & 95.3 & 90.1 & \\
\hline & Essential oil yield\% (w/w) & & & & 0.2 & 0.04 & 0.03 & 0.09 & 0.12 & 0.02 & \\
\hline & Monoterpene hydrocarbons & & & & 61.6 & 52,0 & 54.5 & 58.5 & 57.1 & 1.5 & \\
\hline & Oxygenated monoterpenes & & & & 8.2 & 10.2 & 5.3 & 9.2 & 16.5 & 9.1 & \\
\hline & Sesquiterpene hydrocarbons & & & & 7.2 & 6.6 & 12.2 & 7.5 & 11.4 & 3.8 & \\
\hline & Oxygenated sesquiterpenes & & & & 4.4 & 9.6 & 7,0 & 12.3 & 4.3 & 14.4 & \\
\hline & Phenylpropanoids & & & & 5.4 & 2.2 & 2.5 & 7,0 & 1.9 & 3.3 & \\
\hline & Oxygenated diterpenes & & & & 0.5 & 0.4 & 2.7 & 0.1 & 0.4 & - & \\
\hline & Diterpenes hydrocarbons & & & & 0.1 & 0.2 & 1.7 & 0.1 & 0.1 & 1.3 & \\
\hline & Non-terpenic compounds & & & & 3.5 & 12.5 & 7.7 & 4,0 & 3.6 & 56.7 & \\
\hline
\end{tabular}

a Order of elution is given on apolar column (Rtx-1), b Retention indices of literature on the apolar column (IRla) reported from König et al., 2001.,c Retention indices on the apolar Rtx-1 column (Rla), d Retention indices on the polar Rtx-Wax column (Rlp), e Quantification was carried out using RFs relative to tridecane as internal standard, $\mathrm{g} / 100 \mathrm{~g}$ : concentration expressed in $\mathrm{g} / 100 \mathrm{~g}$ of essential oil are given on the apolar column except for components with identical Rla (concentrations are given on the polar column), $\operatorname{tr}=\operatorname{trace}(<0,05 \mathrm{~g} / 100 \mathrm{~g}), \mathrm{f}$ Rl: Retention Indices; MS: Mass Spectrometry in electronic impact mode; Ref1,: compounds identified from literature data König et al., 2001, Ref2,: compounds identified from literature data NIST Chemistry WebBook.

accompanied by $\alpha$-pinene, which accounted for $8.1 \%$ in stems and always more than $13.1 \%$ in leaves, flowers and umbels. With these hydrocarbon compounds, we noted the occurrence of trans-sabinyl acetate, which had a relatively higher concentration in umbels $(9.6 \%)$ than in the other aerial organs $(2.6 \%-3.8 \%)$. The chemical differences observed between the essential oils and the volatile fractions extracted using HD and SPME, respectively, can be explained by the fact that the first technique is based on the liquid quasi-total extraction of plant volatiles, and the latter technique is controlled by a solid/gas equilibrium step. During hydrodistillation, the most volatile and water soluble compounds are lost in the gaseous phase and in the hydrolate, respectively, whereas, with HS extraction, it is the fiber affinity of each compound that monitors the sampling of the volatiles. As a consequence, it should be noted that 23 compounds (1a-1e, 5a, 8a, 9a, 13a, 14a, 19a, 20a, 21a, 22a, 22b, 27a, 27b, 29a, 38a, 47a, 51a, 54a, and 65a) were only identified in the volatile fractions extracted using HS-SPME.

\section{Antimicrobial activity (assay disk)}

Preliminary screening of the antimicrobial activity in vitro of the essential oils from $D$. muricatus species against nine pathogenic microorganisms were studied using the filter paper disc agar-diffusion technique. The results showed variation in the antimicrobial properties of the plant essential oil (Table 3). The essential oil showed strong activity (inhibition zone $>20 \mathrm{~mm}$ ), moderate activity (inhibition zone $<20-12 \mathrm{~mm}$ ), and no inhibition (zone $<12 \mathrm{~mm}$ ). The highest activity (diameter of inhibition zone $22 \mathrm{~mm}$ ) was demonstrated against S. aureus by the essential oil of the root, while the lowest (diameter of inhibition zone $6 \mathrm{~mm}$ ) was demonstrated against E. coli by oil from the aerial parts. Other hand, C. albicans, B. cereus and L. monocytogenes were also prone to growth inhibition with diameter zones of inhibition ranging from 12 to $16 \mathrm{~mm}$. Rest of the bacterial strains (B. subtilis, E. faecalis, P. aeruginosa, K. pneumonia and E. coli) showed no inhibition, with diameter of zones of inhibition ranging from 8 to $10 \mathrm{~mm}$ (Table 3).

\section{Minimum inhibitory concentrations (MIC)}

The in-vitro antibacterial activities of essential oil from the roots and aerial parts of $D$. muricatus against the employed bacteria were assessed qualitatively and quantitatively by the presence or absence of inhibition zones. The noted antibacterial and antifungal effects of the two are presented in Table 3. In general, the roots oil showed higher activity against bacteria than oil of aerial parts. The most prominent inhibitory action of roots oil was observed against $S$. aureus with a MIC of $0.8 \mu \mathrm{g} / \mathrm{ml}$. However, B. cereus and L. monocytogenes showed moderate activity with MIC values of $65 \mu \mathrm{g} / \mathrm{ml}$. As for the antifungal effect, the aerial parts oil was found to be effective against the pathogenic yeast C. albicans $(\mathrm{MIC}=45 \mu \mathrm{g} / \mathrm{ml})$ and an average activity antimicrobial on the B. cereus and L. monocytogenes with a MIC of $250 \mu \mathrm{g} / \mathrm{ml}$. It should be noted that the highest tested concentration $(6000 \mu \mathrm{g} / \mathrm{ml})$ of had no effect on other growth of microorganisms. Various chemical compounds isolated by hydrodistillation of oils from $D$. muricatus 
Table 2 Chemical composition of $D$. muricatus volatile fractions extracted by HS-SPME

\begin{tabular}{|c|c|c|c|c|c|c|c|c|c|}
\hline \multirow[b]{2}{*}{$\mathbf{N}^{\circ}$} & \multirow[b]{2}{*}{ Components $^{a}$} & \multirow[b]{2}{*}{ IRla b } & \multirow[b]{2}{*}{$\mathrm{Rla}^{\mathrm{C}}$} & \multirow[b]{2}{*}{ Aerial parts } & \multicolumn{5}{|c|}{ Separated organs ${ }^{e}$} \\
\hline & & & & & Stems & Leaves & Flowers & Umbels & Roots \\
\hline $1 a$ & Heptane & 700 & 700 & $2.6 \pm 0.07$ & $7.9 \pm 0.28$ & $1.3 \pm 0.11$ & $1.1 \pm 0.15$ & $0.3 \pm 0.04$ & $0.2 \pm 0.01$ \\
\hline $1 \mathrm{~b}$ & 3-Methyl butanol & 709 & 705 & $0.3 \pm 0.01$ & $0.7 \pm 0.01$ & $0.2 \pm 0.01$ & $0.4 \pm 0.01$ & $0.2 \pm 0.01$ & $0.1 \pm 0.01$ \\
\hline $1 \mathrm{c}$ & 3-Methyl-pentan-2-ol & 754 & 760 & $0.4 \pm 0.14$ & $0.4 \pm 0.01$ & $0.2 \pm 0.01$ & $0.5 \pm 0.09$ & $0.4 \pm 0.01$ & $0.7 \pm 0.01$ \\
\hline $1 d$ & Hexanal & 780 & 771 & $0.2 \pm 0.01$ & $0.4 \pm 0.01$ & $0.1 \pm 0.01$ & $0.1 \pm 0.01$ & $0.3 \pm 0.06$ & $1.4 \pm 0.07$ \\
\hline 1 & Nonane & 900 & 898 & $0.5 \pm 0.07$ & $0.5 \pm 0.02$ & $1.1 \pm 0.22$ & $0.4 \pm 0.01$ & $\operatorname{tr}$ & $0.1 \pm 0.01$ \\
\hline $1 e$ & Artemisiatriene & 923 & 921 & $0.2 \pm 0.01$ & - & - & $0.3 \pm 0.06$ & $0.1 \pm 0.01$ & - \\
\hline 2 & a-Thujene & 932 & 923 & $0.1 \pm 0.01$ & - & $0.1 \pm 0.01$ & $0.5 \pm 0.04$ & $0.1 \pm 0.01$ & - \\
\hline 3 & a-Pinene & 936 & 931 & $13.2 \pm 0.74$ & $8.1 \pm 0.36$ & $16.1 \pm 0.89$ & $13.1 \pm 0.97$ & $15.5 \pm 1.1$ & $8.7 \pm 0.14$ \\
\hline 4 & Camphene & 950 & 944 & $0.2 \pm 0.01$ & $\operatorname{tr}$ & $0.1 \pm 0.01$ & $0.3 \pm 0.01$ & $0.3 \pm 0.08$ & $0.4 \pm 0.01$ \\
\hline $5 a$ & Butyl butyrate & 970 & 966 & $0.2 \pm 0.01$ & - & $0.2 \pm 0.01$ & $0.2 \pm 0.01$ & $0.2 \pm 0.01$ & $0.1 \pm 0.01$ \\
\hline 6 & Sabinene & 973 & 967 & $2.9 \pm 0.21$ & $1.4 \pm 0.14$ & $2.3 \pm 0.45$ & $6.5 \pm 0.33$ & $1.5 \pm 0.46$ & $0.3 \pm 0.01$ \\
\hline 7 & $\beta$-Pinene & 978 & 970 & $0.9 \pm 0.06$ & $0.7 \pm 0.02$ & $1.2 \pm 0.56$ & $1.1 \pm 0.51$ & $1.1 \pm 0.13$ & $1.6 \pm 0.21$ \\
\hline 8 & Myrcene & 987 & 980 & $2.9 \pm 0.13$ & $1.5 \pm 0.14$ & $3.2 \pm 0.21$ & $2.9 \pm 0.12$ & $3.9 \pm 0.66$ & $0.1 \pm 0.01$ \\
\hline $8 a$ & Yomogi alcohol & 991 & 981 & $0.4 \pm 0.07$ & $0.7 \pm 0.01$ & $0.4 \pm 0.01$ & $0.4 \pm 0.01$ & $0.4 \pm 0.05$ & - \\
\hline 9 & a-Phellandrene & 1002 & 997 & $1.5 \pm 0.13$ & - & $3.1 \pm 0.16$ & $2 \pm 0.29$ & $1.1 \pm 0.29$ & - \\
\hline $9 a$ & 3-Carene & 1010 & 1005 & $0.1 \pm 0.01$ & - & $0.1 \pm 0.01$ & $0.1 \pm 0.01$ & $0.1 \pm 0.01$ & $0.4 \pm 0.01$ \\
\hline 10 & a-Terpinene & 1013 & 1008 & $0.8 \pm 0.06$ & - & $0.3 \pm 0.01$ & $2.8 \pm 0.22$ & $0.2 \pm 0.01$ & $0.1 \pm 0.01$ \\
\hline 11 & p-Cymene & 1015 & 1012 & $3.2 \pm 0.32$ & $3.4 \pm 0.12$ & $3.1 \pm 0.51$ & $3.4 \pm 0.51$ & $3.2 \pm 0.11$ & $9.1 \pm 0.76$ \\
\hline 12 & Limonene & 1025 & 1022 & $22.4 \pm 1.28$ & $13.3 \pm 0.56$ & $30.6 \pm 0.99$ & $19.1 \pm 0.77$ & $28.1 \pm 0.82$ & $6.9 \pm 0.35$ \\
\hline 13 & (Z)- $\beta$-Ocimene & 1029 & 1025 & $0.2 \pm 0.01$ & $0.4 \pm 0.01$ & $0.1 \pm 0.01$ & $0.2 \pm 0.02$ & $0.1 \pm 0.01$ & $0.3 \pm 0.01$ \\
\hline $13 a$ & (E)- $\beta$-Ocimene & 1041 & 1031 & $0.1 \pm 0.01$ & $0.1 \pm 0.01$ & $0.2 \pm 0.02$ & $0.2 \pm 0.01$ & $0.1 \pm 0.01$ & $0.3 \pm 0.01$ \\
\hline 14 & $\mathrm{Y}$-Terpinene & 1051 & 1049 & $3.2 \pm 0.09$ & $4.1 \pm 0.54$ & $2.5 \pm 0.22$ & $5.1 \pm 0.55$ & $1.3 \pm 0.35$ & $1.2 \pm 0.01$ \\
\hline $14 a$ & Octanol & 1063 & 1052 & $0.3 \pm 0.01$ & $\operatorname{tr}$ & $0.6 \pm 0.01$ & $0.4 \pm 0.08$ & $0.3 \pm 0.05$ & - \\
\hline 16 & Nonan-2-one & 1070 & 1073 & $0.6 \pm 0.07$ & $0.7 \pm 0.02$ & $0.5 \pm 0.01$ & $0.6 \pm 0.08$ & $0.6 \pm 0.09$ & $0.2 \pm 0.01$ \\
\hline 17 & p-Cymenene & 1075 & 1077 & $0.3 \pm 0.06$ & - & $0.2 \pm 0.01$ & $0.4 \pm 0.06$ & $0.7 \pm 0.03$ & $3.1 \pm 0.13$ \\
\hline 18 & Terpinolene & 1082 & 1079 & $0.7 \pm 0.06$ & $0.6 \pm 0.01$ & $0.9 \pm 0.07$ & $1.3 \pm 0.23$ & $0.4 \pm 0.01$ & $10.2 \pm 0.86$ \\
\hline 19 & Linalool & 1083 & 1085 & $1.4 \pm 0.08$ & $3.2 \pm 0.28$ & $0.5 \pm 0.01$ & $1.1 \pm 0.29$ & $0.9 \pm 0.1$ & $0.2 \pm 0.01$ \\
\hline $19 a$ & a-Thujone & 1089 & 1086 & $0.5 \pm 0.04$ & $1.8 \pm 0.09$ & $\operatorname{tr}$ & $0.5 \pm 0.02$ & $0.4 \pm 0.02$ & $0.1 \pm 0.01$ \\
\hline 20 & Undecane & 1100 & 1100 & $8.9 \pm 0.76$ & $16.9 \pm 0.89$ & $6.1 \pm 0.38$ & $3.6 \pm 0.14$ & $7.4 \pm 0.69$ & $7.2 \pm 0.26$ \\
\hline $20 a$ & 3-Octyl acetate & 1113 & 1103 & $1.8 \pm 0.12$ & $0.7 \pm 0.06$ & $0.2 \pm 0.01$ & $0.3 \pm 0.01$ & $0.2 \pm 0.01$ & $0.2 \pm 0.01$ \\
\hline $21 a$ & Camphor & 1123 & 1119 & $1.1 \pm 0.07$ & $2.9 \pm 0.16$ & $0.7 \pm 0.01$ & $0.6 \pm 0.06$ & $0.2 \pm 0.01$ & $0.5 \pm 0.01$ \\
\hline 22 & trans-Pinocarveol & 1126 & 1120 & $0.4 \pm 0.01$ & $0.7 \pm 0.01$ & $0.3 \pm 0.02$ & $0.4 \pm 0.01$ & $0.6 \pm 0.02$ & $0.1 \pm 0.01$ \\
\hline $22 a$ & Citronellal & 1129 & 1130 & $0.1 \pm 0.01$ & $\operatorname{tr}$ & $0.1 \pm 0.01$ & $0.1 \pm 0.01$ & $0.4 \pm 0.05$ & - \\
\hline $22 b$ & cis-Verbenol & 1132 & 1132 & $0.1 \pm 0.01$ & - & $0.1 \pm 0.01$ & $0.1 \pm 0.01$ & $0.1 \pm 0.01$ & - \\
\hline 24 & Pinocarvone & 1137 & 1135 & $0.1 \pm 0.01$ & - & $0.1 \pm 0.01$ & $0.1 \pm 0.01$ & $0.2 \pm 0.02$ & $0.7 \pm 0.01$ \\
\hline 25 & Borneol & 1150 & 1149 & $0.5 \pm 0.08$ & $0.9 \pm 0.01$ & $0.4 \pm 0.01$ & $0.3 \pm 0.04$ & $0.4 \pm 0.01$ & - \\
\hline 26 & Cryptone & 1160 & 1159 & $0.5 \pm 0.06$ & $0.5 \pm 0.01$ & $0.5 \pm 0.08$ & $0.4 \pm 0.01$ & $0.7 \pm 0.09$ & $3.8 \pm 0.11$ \\
\hline 27 & Terpinene-4-ol & 1164 & 1160 & $1.4 \pm 0.18$ & $2.1 \pm 0.43$ & $0.9 \pm 0.06$ & $1.8 \pm 0.36$ & $1.1 \pm 0.15$ & $0.1 \pm 0.01$ \\
\hline $27 a$ & Myrtenal & 1172 & 1163 & $0.1 \pm 0.01$ & $\operatorname{tr}$ & $0.1 \pm 0.01$ & $0.1 \pm 0.01$ & $0.1 \pm 0.01$ & $0.3 \pm 0.02$ \\
\hline $27 \mathrm{~b}$ & Estragole & 1175 & 1169 & $1.1 \pm 0.09$ & $1.1 \pm 0.58$ & $1.3 \pm 0.29$ & $1.7 \pm 0.12$ & $0.6 \pm 0.08$ & - \\
\hline 29 & a-Terpineol & 1176 & 1177 & $0.4 \pm 0.05$ & $0.5 \pm 0.04$ & $0.4 \pm 0.08$ & $0.4 \pm 0.03$ & $0.5 \pm 0.01$ & $0.2 \pm 0.01$ \\
\hline $29 a$ & Verbenone & 1184 & 1178 & $0.3 \pm 0.01$ & $0.7 \pm 0.01$ & $0.1 \pm 0.01$ & $0.2 \pm 0.01$ & $0.1 \pm 0.01$ & - \\
\hline 31 & Decanal & 1188 & 1185 & $0.1 \pm 0.01$ & - & $0.1 \pm 0.01$ & $0.1 \pm 0.01$ & $0.2 \pm 0.01$ & - \\
\hline 33 & Citronellol & 1213 & 1211 & $1.1 \pm 0.14$ & $1.1 \pm 0.22$ & $1.1 \pm 0.33$ & $0.9 \pm 0.06$ & $1.5 \pm 0.23$ & $0.1 \pm 0.01$ \\
\hline 34 & Carvone & 1214 & 1215 & $0.7 \pm 0.07$ & $1.1 \pm 0.09$ & $0.5 \pm 0.01$ & $0.6 \pm 0.04$ & $0.6 \pm 0.02$ & $0.1 \pm 0.01$ \\
\hline 35 & Pulegone & 1215 & 1216 & $0.3 \pm 0.01$ & $0.8 \pm 0.02$ & $0.2 \pm 0.01$ & $0.2 \pm 0.01$ & $0.1 \pm 0.01$ & - \\
\hline
\end{tabular}


Table 2 Chemical composition of $D$. muricatus volatile fractions extracted by HS-SPME (Continued)

\begin{tabular}{|c|c|c|c|c|c|c|c|c|c|}
\hline 37 & Geraniol & 1235 & 1235 & $0.2 \pm 0.01$ & $\operatorname{tr}$ & $0.1 \pm 0.01$ & $0.4 \pm 0.02$ & $0.1 \pm 0.01$ & $0.8 \pm 0.05$ \\
\hline 38 & trans-Myrtanol & 1240 & 1236 & $0.1 \pm 0.01$ & $0.1 \pm 0.01$ & $\operatorname{tr}$ & $0.1 \pm 0.01$ & $0.3 \pm 0.01$ & - \\
\hline $38 a$ & Geranial & 1244 & 1239 & $0.1 \pm 0.01$ & $\operatorname{tr}$ & $0.1 \pm 0.01$ & $0.1 \pm 0.01$ & $\operatorname{tr}$ & - \\
\hline 39 & cis-Chrysanthenyl acetate & 1253 & 1242 & $0.1 \pm 0.01$ & $\operatorname{tr}$ & $0.1 \pm 0.01$ & $\operatorname{tr}$ & $0.1 \pm 0.01$ & - \\
\hline 42 & Bornyl acetate & 1270 & 1266 & $0.6 \pm 0.05$ & $1.2 \pm 0.11$ & $0.4 \pm 0.05$ & $0.5 \pm 0.06$ & $0.6 \pm 0.04$ & $9.7 \pm 0.55$ \\
\hline 43 & Undecan-2-one & 1273 & 1270 & $0.6 \pm 0.08$ & $0.8 \pm 0.04$ & $0.6 \pm 0.08$ & $0.4 \pm 0.03$ & $0.5 \pm 0.01$ & $0.3 \pm 0.01$ \\
\hline 44 & trans-Sabinyl acetate & 1278 & 1271 & $5.1 \pm 0.47$ & $3.8 \pm 0.26$ & $2.6 \pm 0.12$ & $3.5 \pm 0.38$ & $9.6 \pm 0.53$ & \\
\hline $47 a$ & Neryl acetate & 1342 & 1335 & $2.0 \pm 0.31$ & $2.4 \pm 0.36$ & $3.4 \pm 0.65$ & $1.3 \pm 0.35$ & $0.9 \pm 0.1$ & $0.3 \pm 0.01$ \\
\hline 48 & 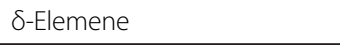 & 1340 & 1337 & $0.3 \pm 0.02$ & $0.7 \pm 0.06$ & $0.3 \pm 0.05$ & $0.1 \pm 0.01$ & $\mathrm{tr}$ & $0.1 \pm 0.01$ \\
\hline 49 & Geranyl acetate & 1362 & 1360 & $0.1 \pm 0.01$ & $\operatorname{tr}$ & tr & $0.2 \pm 0.01$ & $\operatorname{tr}$ & $0.2 \pm 0.01$ \\
\hline 50 & Undecanol & 1363 & 1365 & $0.1 \pm 0.01$ & $\operatorname{tr}$ & $\operatorname{tr}$ & $\operatorname{tr}$ & $\operatorname{tr}$ & $1.9 \pm 0.01$ \\
\hline 51 & a-Copaene & 1379 & 1377 & $0.7 \pm 0.01$ & $1.1 \pm 0.12$ & $0.4 \pm 0.02$ & $0.6 \pm 0.03$ & $0.9 \pm 0.08$ & $0.4 \pm 0.01$ \\
\hline $51 a$ & Daucene & 1380 & 1379 & $0.5 \pm 0.02$ & $0.9 \pm 0.08$ & $0.1 \pm 0.01$ & $0.2 \pm 0.01$ & $0.7 \pm 0.01$ & $0.7 \pm 0.06$ \\
\hline 54 & Dodecanal & 1389 & 1395 & $0.2 \pm 0.01$ & $0.3 \pm 0.01$ & $0.1 \pm 0.01$ & $0.1 \pm 0.01$ & $0.1 \pm 0.01$ & $1.3 \pm 0.01$ \\
\hline $54 a$ & Tetradecane & 1400 & 1399 & $0.1 \pm 0.01$ & $0.2 \pm 0.02$ & $0.1 \pm 0.01$ & $0.1 \pm 0.01$ & $\operatorname{tr}$ & - \\
\hline 56 & trans-Caryophyllene & 1424 & 1422 & $2.3 \pm 0.23$ & $1.4 \pm 0.13$ & $3.1 \pm 0.25$ & $3.8 \pm 0.26$ & $1.2 \pm 0.15$ & $1.9 \pm 0.18$ \\
\hline 58 & $\beta$-Copaene & 1430 & 1430 & $0.3 \pm 0.02$ & $0.4 \pm 0.01$ & $0.6 \pm 0.05$ & $0.3 \pm 0.01$ & $0.3 \pm 0.01$ & $0.4 \pm 0.01$ \\
\hline 59 & a-Humulene & 1455 & 1450 & $0.4 \pm 0.05$ & - & $0.1 \pm 0.01$ & $0.9 \pm 0.08$ & $0.1 \pm 0.01$ & $0.1 \pm 0.01$ \\
\hline 61 & Dodecanol & 1472 & 1468 & $\operatorname{tr}$ & tr & tr & $\mathrm{tr}$ & $\mathrm{tr}$ & $3.4 \pm 0.28$ \\
\hline 62 & Y-Muurolene & 1473 & 1471 & $1.3 \pm 0.13$ & $0.5 \pm 0.01$ & $0.6 \pm 0.01$ & $1.5 \pm 0.42$ & $1.9 \pm 0.21$ & $4.3 \pm 0.16$ \\
\hline 63 & Germacrene D & 1479 & 1476 & $0.4 \pm 0.04$ & $0.5 \pm 0.06$ & $0.2 \pm 0.01$ & $0.4 \pm 0.02$ & $0.3 \pm 0.01$ & $0.2 \pm 0.01$ \\
\hline 64 & trans- $\beta$-Bergamotene & 1480 & 1475 & $\operatorname{tr}$ & $\operatorname{tr}$ & tr & $\operatorname{tr}$ & $\operatorname{tr}$ & $0.5 \pm 0.03$ \\
\hline 65 & 6-epi-Shyobunone & 1481 & 1480 & $0.1 \pm 0.01$ & $\mathrm{tr}$ & $0.1 \pm 0.01$ & $0.2 \pm 0.01$ & $0.2 \pm 0.01$ & - \\
\hline $65 a$ & $\beta$-Selinene & 1486 & 1481 & $0.2 \pm 0.01$ & $0.2 \pm 0.01$ & $0.2 \pm 0.02$ & $0.1 \pm 0.01$ & $0.2 \pm 0.03$ & $0.5 \pm 0.02$ \\
\hline 69 & Shyobunone & 1500 & 1501 & $0.1 \pm 0.01$ & $\operatorname{tr}$ & $0.1 \pm 0.01$ & $0.1 \pm 0.01$ & $0.1 \pm 0.01$ & $0.7 \pm 0.01$ \\
\hline 70 & $\delta$-Cadinene & 1520 & 1515 & $0.6 \pm 0.04$ & $0.5 \pm 0.01$ & $0.3 \pm 0.04$ & $0.1 \pm 0.01$ & $1.3 \pm 0.16$ & $0.9 \pm 0.07$ \\
\hline 71 & (E)-a-Bisabolene & 1531 & 1526 & $1.1 \pm 0.31$ & $0.5 \pm 0.02$ & $1.4 \pm 0.12$ & $0.9 \pm 0.1$ & $1 \pm 0.45$ & $0.4 \pm 0.01$ \\
\hline 72 & Isochavicol isobutyrate & 1541 & 1538 & $0.3 \pm 0.05$ & $\operatorname{tr}$ & $0.3 \pm 0.01$ & $0.9 \pm 0.06$ & $0.1 \pm 0.01$ & $0.1 \pm 0.1$ \\
\hline 76 & Caryophyllene oxide & 1578 & 1582 & $0.2 \pm 0.01$ & - & $0.1 \pm 0.01$ & $0.4 \pm 0.01$ & $0.1 \pm 0.01$ & $0.7 \pm 0.01$ \\
\hline 77 & Tridecanol & 1580 & 1586 & $\operatorname{tr}$ & $\mathrm{tr}$ & $\operatorname{tr}$ & $\operatorname{tr}$ & $\operatorname{tr}$ & $1.1 \pm 0.07$ \\
\hline 87 & Eudesma-4(15),7-dien-1 $\beta$-ol & 1671 & 1672 & $\operatorname{tr}$ & - & tr & $\operatorname{tr}$ & $\operatorname{tr}$ & $0.6 \pm 0.01$ \\
\hline \multirow[t]{9}{*}{89} & Heptadecane & 1700 & 1699 & $0.4 \pm 0.13$ & - & $1.3 \pm 0.65$ & $0.2 \pm 0.01$ & $0.2 \pm 0.01$ & - \\
\hline & Total identification (\%) & & & 97.8 & 95.4 & 99.1 & 94.3 & 97.6 & 90.6 \\
\hline & GC-FID Total area $10^{5}$ & & & 103 & 22 & 154 & 108 & 107 & 68 \\
\hline & Monoterpene hydrocarbons & & & 52.9 & 33.6 & 64.1 & 59.3 & 57.8 & 42.8 \\
\hline & Oxygenated monoterpenes & & & 20.6 & 25.6 & 14.4 & 16 & 20.5 & 17.6 \\
\hline & Sesquiterpene hydrocarbons & & & 8.1 & 5.8 & 7.2 & 8.7 & 7.2 & 10.4 \\
\hline & Oxygenated sesquiterpenes & & & 0.4 & - & 0.3 & 0.7 & 0.4 & 2.0 \\
\hline & Phenylpropanoids & & & 0.3 & - & 0.3 & 0.9 & 0.1 & 0.1 \\
\hline & Non-terpenic compounds & & & 15.5 & 30.4 & 12.8 & 8.7 & 11.6 & 17.7 \\
\hline
\end{tabular}

a Order of elution is given on apolar column (Rtx-1). Numbers correspond to those in Table 1. The volatile components identified exclusively from the $\mathrm{HS}$-fractions were affected by a letter, b Retention indices of literature on the apolar column (IRla) reported from König et al., 2001., c Retention indices on the apolar Rtx-1 column (Rla), d Percentages (means of three analyses) obtained by GC-FID (on RTX-1: apolar column) under optimized HS-SPME parameters: temperature: $70^{\circ} \mathrm{C}$, equilibrium time: $120 \mathrm{~min}$; extraction time: $30 \mathrm{~min}$.

have direct activity against many species of bacteria, such as terpenes and a variety of aliphatic hydrocarbons (alcohols, aldehydes and ketones). The lipophilic character of their hydrocarbon skeleton and the hydrophilic character of their functional groups are of main importance in the antimicrobial action of essential oils components. Therefore, a rank of activity has been proposed as follows: phenols $>$ aldehydes $>$ ketones $>$ alcohols $>$ esters $>$ hydrocarbons [28]. The activity of the roots oil could be explained at least partially by its 
Table 3 Antimicrobial activity of $D$. muricatus essential oil

\begin{tabular}{|c|c|c|c|c|}
\hline \multirow[t]{2}{*}{ Microorganisms } & \multicolumn{2}{|c|}{$\begin{array}{l}\text { Disc diffusion } \\
\text { assay }(\mathrm{mm})\end{array}$} & \multicolumn{2}{|c|}{ MIC $(\mu \mathrm{g} / \mathrm{ml})$} \\
\hline & Roots & Aerial parts & Roots & Aerial parts \\
\hline \multicolumn{5}{|c|}{ Gram-positive bacterium } \\
\hline B. subtilis & 9 & 8 & $>6000$ & $>6000$ \\
\hline L. monocytogenes & 14 & 13 & 65 & 250 \\
\hline B. cereus & 15 & 12 & 65 & 250 \\
\hline S. aureus & 22 & 10 & 8 & $>6000$ \\
\hline$P$. aeruginosa & 9 & 10 & $>6000$ & $>6000$ \\
\hline E. faecalis & 10 & 8 & $>6000$ & $>6000$ \\
\hline \multicolumn{5}{|c|}{ Gram-negative bacterium } \\
\hline K. pneumoniae & 9 & 8 & $>6000$ & $>6000$ \\
\hline E. coli & 8 & 6 & $>6000$ & $>6000$ \\
\hline \multicolumn{5}{|l|}{ Yeast } \\
\hline C. albicans & 12 & 16 & 95 & 45 \\
\hline
\end{tabular}

content of undecan-2-one (10.2\%). This ketone was previously proved to have antimicrobial and nematicidal activity $[29,30]$. The higher activity of the roots oil compared to the aerial parts oil could be attributed to this fact. Another major class of this oil, aliphatic alcohols was, likewise, previously reported as an antimicrobial compound and was reported to possess strong to moderate activities against several bacteria [31].

\section{Conclusion}

Volatiles isolated from separated organs of $D$. muricatus by HS-SPME and hydrodistillation were investigated using GC-RI and GC-MS. Concerning the essential oils, oil from $D$. muricatus roots was mainly composed of oxygenated compounds, while oil from aerial parts (i.e., the leaves, stems, flowers, and umbels) was dominated by hydrocarbon compounds. Moreover, the study of the volatiles sampled by HS-SPME showed that the chemical composition of the HS fractions obtained from different organs was qualitatively similar but differed by the relative concentrations of the main components. It is interesting to note that the sample preparation method impacted quantitatively on the GC profile of $D$. muricatus volatiles. The antimicrobial properties of $D$. muricatus essential oils tested on nine microorganisms species showed that oil from roots was active against $S$. aureus, while essential oil obtained from aerial parts was active against the yeast $C$. albicans.

\section{Experimental}

\section{Plant material and oil isolation}

Separated organs (stems, leaves, flowers, umbels and roots) from $D$. muricatus were collected in Bensekrane forest area (North West of Tlemcen, Algeria) [287 m, $\left.35{ }^{\circ} 07^{\prime} \mathrm{N} 1{ }^{\circ} 22^{\prime} \mathrm{O}\right]$ on September 2009. Voucher specimens were deposited in the herbarium of the Tlemcen University Botanical Laboratory (Voucher number: UBL 128.09). A portion of each organ was stored at $4^{\circ} \mathrm{C}$ for eventual further studies. The oils were isolated by hydrodistillation (400-450 $\mathrm{g}$ of plant per sample) for $6 \mathrm{~h}$ using a Clevenger-type apparatus [32] according to the European Pharmacopoeia and yielded $0.02 \%$ for roots and $0.03-0.12 \%$ for aerial parts $\mathrm{w} / \mathrm{w}$ of oil.

\section{HS-SPME conditions}

The single organs of $D$. muricatus (stems, leaves, flowers, umbels and roots separately) were cut roughly with scissors (1-2 cm long) before subjection to HS-SPME. The SPME device (Supelco) coated with divinylbenzene/ carboxen/polydimethylsiloxane (DVB/CAR/PDMS, $30 \mu \mathrm{m}$ ) was used for extraction of the plant volatiles. Optimization of conditions was carried out using fresh organs of the plant (1 g in a $20 \mathrm{~mL}$ vial) and based on the number and the sum of total peak areas measured on GC-FID. Temperature, equilibration time and extraction time were selected after nine experiments combining four temperatures (30, 50, 70 and $90^{\circ} \mathrm{C}$ ), four equilibration times (20, 40, 60 and $80 \mathrm{~min})$ and three extraction times (15, 30 and $45 \mathrm{~min})$. After sampling, SPME fibre was inserted into the GC and GC-MS injection ports for desorption of volatile components (5 min), both using the splitless injection mode. Before sampling, each fibre was reconditioned for $5 \mathrm{~min}$ in the GC injection port at $260^{\circ} \mathrm{C}$. HS-SPME and subsequent analyses were performed in triplicate. The coefficient of variation $(1.6 \%<\mathrm{CV}<17.8 \%)$ calculated on the basis of total area obtained from the FID-signal for the samples indicated that the HS-SPME method produced reliable results.

\section{Gas chromatography}

GC analyses were carried out using a Perkin-Elmer (Waltham, MA, USA) Autosystem XL GC apparatus equipped with a dual flame ionization detection system and a fused-silica capillary columns (60 m x $0.22 \mathrm{~mm}$ I.D., film thickness $0.25 \mu \mathrm{m}$ ), Rtx-1 (polydimethylsiloxane). The oven temperature was programmed from $60^{\circ} \mathrm{C}$ to $230^{\circ} \mathrm{C}$ at $2^{\circ} \mathrm{C} / \mathrm{min}$ and then held isothermally at $230^{\circ} \mathrm{C}$ for $35 \mathrm{~min}$. Injector and detector temperatures were maintained at $280^{\circ} \mathrm{C}$. Samples were injected in the split mode $(1 / 50)$, using helium as the carrier gas $(1 \mathrm{~mL} / \mathrm{min})$; the injection volume was $0.2 \mu \mathrm{L}$. Retention indices (RI) of the compounds were determined from a software from PerkinElmer. Component relative concentrations were calculated based on GC peak areas without using correction factors.

\section{Gas chromatography-mass spectrometry}

Samples were analyzed with a Perkin-Elmer Turbo mass detector (quadrupole), coupled to a Perkin-Elmer Autosystem XL, equipped with the fused-silica capillary 
columns Rtx-1 and Rtx-Wax (ion source temperature $150^{\circ} \mathrm{C}$; energy ionization $70 \mathrm{eV}$ ). EI mass spectra were acquired over the mass range 35-350 Da (scan time: $1 \mathrm{~s})$. Other GC conditions were the same as described under GC except split 1/80.

\section{Component identification}

Identification of the components was based (i) on the comparison of their GC retention indices (RI) on non polar and polar columns, determined relative to the retention time of a series of $\mathrm{n}$-alkanes with linear interpolation, with those of authentic compounds or literature data [33,34]; and (ii) on computer matching with commercial mass spectral libraries [33-35] and comparison of spectra with those of our personal library.

\section{Component quantification}

Quantification of essential oil components was expressed using relative concentration in $\mathrm{g} / 100 \mathrm{~g}$ of essential oil. The procedure included the calcul of FID response factors (RFs) relative to an internal standard. We carried out a methodology reported in the literature [36] and improved in our laboratory [37]. The application of this analytical procedure allowed the determination of the oil component relative concentrations expressed in $\mathrm{g} / 100 \mathrm{~g}$ of essential oil. Relative amounts of individual components obtained during HS-SPME experiments, were calculated on the basis of their GC peak areas on the Rtx-1 capillary column, without FID response factor correction.

\section{Bacterial and yeast strains and media}

The bacterial strains used in this study, i.e. Staphylococcus aureus, Bacillus subtilis, Pseudomonas aeruginosa, Enterococcus faecalis, Listeria monocytogenes, Bacillus cereus (Gram positive), Escherichia coli and Klebsiella pneumoniae (gram negative) were isolated at the Medical Reanimation Department of the Hospital University Center of Tlemcen in Algeria. The yeast Candida albicans was isolated at the Dermatology Department of the same hospital. Bacterial strains preserved in nutrient agar at $4^{\circ} \mathrm{C}$, were revivified in nutrient solution and incubated at $37 \pm 1^{\circ} \mathrm{C}$ during 18 to $24 \mathrm{~h} .0 .1 \mathrm{~mL}$ of each culture was added to $10 \mathrm{~mL}$ BHIB (Brain Heart Infusion Broth, pronadisa Hispanalab). C. albicans preserved at $4^{\circ} \mathrm{C}$ in the Sabouraud agar supplemented with chloramphenicol was revivified in nutrient solution and incubated at $30 \pm 1^{\circ} \mathrm{C}$ during 24 to 48 h. $0.1 \mathrm{~mL}$ of each culture was added to $10 \mathrm{~mL}$ sterile physiological water. For antimicrobial assay, bacterial strains were grown on Mueller-Hinton Agar (MHA, Pronadisa Hispanalab) while C. albicans was grown on Sabouraud Dextrose Agar + chloramphenicol (SDA, Merck). Bacterial and yeast inoculate reached microbial densities in the range 106 to $107 \mathrm{cfu} / \mathrm{mL}$.

\section{Antimicrobial activity}

\section{Paper-disc diffusion method}

Antibacterial activities of essential oil from root and all aerial parts of the plant were assessed using the paper disk agar diffusion method according to Rios [38]. Absorbent disks (Whatman disk 6-mm diameter) were impregnated with $20 \mu \mathrm{l}$ of oil, to concentration of $5 \mathrm{mg} \mathrm{mL}-1$, and then placed on the surface of inoculated plates $(90 \mathrm{~mm})$ and incubated at $37^{\circ} \mathrm{C}$ for $24 \mathrm{~h}$. Negative controls were prepared using a disk impregnated with the same solvent as that used to dissolve the plant oils. Antimicrobial activity was assessed by measuring the inhibition zone. All the tests were performed in triplicate.

\section{Dilution-agar method}

A dilution agar method was used to determine the Minimum Inhibitory Concentrations (MIC). Stock solutions were obtained by dissolving extracts in dimethylsulfoxide (DMSO 1\%). Serial dilutions were made to obtain concentrations ranging from 0 to $100 \mu \mathrm{g} \mathrm{mL}-1$ of the essential oil. Each mixture was added to Mueller-Hinton agar for bacteria [39,40]. The Petri dishes contained a sterile solution of DMSO and the culture medium, respectively. After incubation at $37^{\circ} \mathrm{C}$ for $24 \mathrm{~h}$ for bacteria and at $30^{\circ} \mathrm{C}$ for $48 \mathrm{~h}$ for the yeast. The experiments were performed in triplicate.

\section{Competing interest}

The authors declare that they have no competing interests.

\section{Authors' contributions}

MAD performed in collection of plant material. MAD, ND and JMD performed the HD and HS-SPME extractions, obtained the essential oils and the volatiles fractions, as well as participated in the data analysis. MAD, HA, BT, AM and JC conceived the study and helped draft the manuscript. HA, BT, $\mathrm{AM}$ and JC performed the coordination of the study, worked on the data analysis and interpretation. All authors read and approved the final manuscript.

\section{Acknowledgments}

The authors are grateful to Prof. M. Bouazza (Botanical Laboratory, Biology Department, Aboubekr Belkaïd University) for the identification of the vegetable matter, and are indebted to the Agence Universitaire de la Francophonie (AUF) for providing a research grand of N.D., and the Ministère des Affaires Etrangères et Européennes throughout the research program "Partenariat Hubert Curien Tassili".

\section{Author details}

'Laboratoire des Substances Naturelles et Bioactives, Université de Tlemcen, BP 119, 13000, Tlemcen, Algérie. ²UMR CNRS 6134, Laboratoire Chimie des Produits Naturels, Campus Grimaldi, Université de Corse, BP 52, 20250, Corte, France.

Received: 27 January 2012 Accepted: 26 April 2012

Published: 30 May 2012

\section{References}

1. Quézel P, Santa S: Nouvelle Flore de l'Algérie et des Régions Désertiques Méridionales 1962-1963. Paris: Éditions du Centre National de la Recherche Scientifique

2. Sáenz Laín C: Research on Daucus L. (Umbelliferae). Anales Jard Bot Madrid 1981, 37:480-533. 
3. Kula J, Izydorczyk K, Czajkowska A, Bonikowski R: Chemical composition of carrot umbells oils from Daucus carota ssp. sativus cultivated in Poland. Flavour Fragr J 2006, 21:667-669.

4. Saad HEA, ElSharkawy SH, Halim AF: Essential oils of Daucus carota ssp. maximus. Pharm Acta Helv 1995, 70:79-84

5. Flamini G, Cioni PL, Maccioni S, Baldini R: Composition of the essential oil of Daucus gingidium L. ssp. gingidium. Food Chem 2003, 103:1237-1240.

6. Jabrane A, Ben Jannet H, Harzallah-Skhiri F, Mastouri M, Casanova J, Mighri Z: Flower and Root Oils of the Tunisian Daucus carota L. ssp. maritimus (Apiaceae): Integrated Analyses by GC, GC/MS, and 13C-NMR Spectroscopy, and in vitro Antibacterial Activity. Chem Biodivers 2009, 6:881-889.

7. Radulovic N, Đord-evic N, Stojanovic'-Radic' Z: Volatiles of the Balkan endemic Daucus guttatus ssp. zahariadii and cultivated and wildgrowing D. carota - A comparison study. Food Chem 2011, 125:35-43.

8. Mazzoni V, Tomi F, Casanova J: A daucane-type sesquiterpene from Daucus carota seed oil. Flavour Fragr J 1999, 14:268-272

9. Mockute D, Nivinskiene O: The sabinene chemotype of essential oil of seeds of Daucus carota L. ssp. carota growing wild in Lithuania. J Essent Oil Res 2004, 16:277-281

10. Staniszewska M, Kula J, Wieczorkiewicz M, Kusewicz D: Essential oils of wild and cultivated carrots-the chemical composition and antimicrobial activity. J Essent Oil Res 2005, 17:579-583.

11. Wu Y, Xu ZL, Li HJ, Meng XY, Bao YL, Li YX: Components of essential oils in different parts of Daucus carota L. Chem Res Chin Univ 2006, 22:328-334.

12. Rossi PG, Bao L, Luciani A, Panighi J, Desjobert JM, Costa J, Casanova J, Bolla JM, Berti L: (E)-Methylisoeugenol and elemicin: antibacterial components of Daucus carota L. essential oils against Campylobacter jejuni. J Agric Food Chem 2007, 55:7332-7336.

13. Castroviejo S: Flora Ibérica. Madrid: CSIC; 2003. vol. X: Real Jardin Botánico.

14. Danute $M$, Ona N: The Sabinene Chemotype of Essential Oil of Seeds of Daucus carota L. ssp. carota Growing Wild in Lithuania. J Essent Oil Res 2004, 16:277-281.

15. Gonny M, Bradesi P, Casanova J: Identification of the components of the essential oil from wild Corsican Daucus carota L. using 13C-NMR spectroscopy. Flavour Fragr J 2004, 19:424-433.

16. Staniszewska M, Kula J: Composition of the essential oil from wild carrot. L. ssp. carota) growing in Poland. J Essent Oil Res 2001, 13:439-441.

17. Djarri L, Medjroubi K, Akkal S, Elomri A, Seguin E, Vérité P: Composition of the essential oil of aerial parts of an endemic species of the Apiaceae of Algeria, Daucus reboudii Coss. Flavour Fragr J 2006, 21:647-649.

18. Dib MA, Djabou N, Desjobert JM, Allali H, Tabti B, Muselli A, Costa J: Characterization of volatile compounds of Daucus crinitus Desf. Headspace Solid Phase Microextraction as alternative technique to Hydrodistillation. Chem Central J 2010, 4:16.

19. Lanfranchi DA, Laouer H, El Kolli M, Prado S, Maulay-Bailly C, Baldovini N: Bioactive Phenylpropanoids from Daucus crinitus Desf. from Algeria. J Agric Food Chem 2008, 58:2174-2179.

20. Kilibarda V, Nanusevic N, Dogovic N, Ivanic R, Savin K: Content of the essential oil of the carrot and its antibacterial activity. Pharmazie 1996 51:777-778

21. Smaili T, Zellagui A, Cioni P-L, Flamini G: A Myristicin-rich Essential Oi from Daucus sahariensis Growing in Algeria. Nat Prod Comm 2011, 6:883-886.

22. Marzouki $H$, Khaldi A, Falconieri D, Piras A, Marongiu B, Molicotti $P$, Zanetti S: Essential Oils of Daucus carota subsp. carota of Tunisia Obtained by Supercritical Carbon Dioxide Extraction. Nat Prod Comm 2010, 5:1955-1958

23. Chizzola R: Composition of the Essential Oil from Daucus carota ssp carota Growing Wild in Vienna. J Essent Oil Bear Plant 2010, 13:12-19.

24. Baser Kemal HC, Kurkcuoglu M, Askun T, Tumen G: Anti-tuberculosis Activity of Daucus littoralis Sibth. et Sm. (Apiaceae) From Turkey. J Essent Oil Res 2009, 21:572-575.

25. Maxia A, Marongiu B, Piras A, Porcedda S, Tuveri E, Gonçalves MJ, Cavaleiro C, Salgueiro L: Chemical characterization and biological activity of essential oils from Daucus carota L. subsp carota growing wild on the Mediterranean coast and on the Atlantic coast. Fitoterapia 2009, 80:57-61.

26. Tavares AC, Goncalves MJ, Cavaleiro C, Cruz MT, Lopes MC, Canhoto J, Salgueiro L: Essential oil of Daucus carota subsp halophilus: Composition, antifungal activity and cytotoxicity. J Ethnopharmacol 2008, 119:129-134.
27. Mojab F, Hamedi A, Nickavar B, Javidnia K, Shaheed B: Hydrodistilled Volatile Constituents of the Leaves of Daucus carota L. subsp. sativus (Hoffman.)Arcang. (Apiaceae) from Iran. J Essent Oil Bear Plant 2008, 11:271-277.

28. Kalemba D, Kunicka A: Antibacterial and antifungal properties of essential oils. Curr Med Chem 2003, 10:813.

29. Benhadj FM, Marzouk B, Chraif I, Boukef K: Analysis of tunisian Ruta graveolens L. oils from Jemmel. J Food Agr Environ 2007, 5:52-55.

30. Nikoletta G, Francesca N, Manconi, Leonti M, Maxia A, Caboni P: Aliphatic Ketones from Ruta chalepensis (Rutaceae) Induce Paralysis on Root Knot Nematodes. J Agric Food Chem 2011, 59:322-332.

31. Kabelitz N, Santos PM, Heipieper HJ: Effect of aliphatic alcohols on growth and degree of saturation of membrane lipids in Acinetobacter calcoaceticus. FEMS Microbiol Lett 2003, 220:223-227.

32. Conseil de l'Europe: Pharmacopée Européenne, Maisonneuve S.A.. Sainte Ruffine. 1996.

33. Terpenoids and Related Constituents of Essential Oils. Hamburg: Library of MassFinder 2.1 University of Hamburg, Institute of Organic Chemistry; 2001.

34. National Institute of Standards and Technology: NIST Chemistry WebBook. Gaisthersburg, MD: NIST Standard Reference Database; 2005 [http:// webbook.nist.gov/chemistry].

35. Adams RP: Identification of essential oil components by gas chromatography/ quadrupole mass spectroscopy. Carol Stream, IL, USA: Allured Publishing; 2001.

36. Costa R, Zellner BA, Crupi ML, Fina M, Valentino M, Dugo M, Dugo G, Mondello L: Univ Messina, Fac Farm, Dipt Farm-Chim, Viale Annunziata, IT-98168 Messina, Italy. GC-MS, GC-O and enantio-GC investigation of the essential oil of Tarchonanthus camphoratus L. Flavour Fragr J 2008, 23:40-48.

37. Djabou N, Paolini J, Desjobert JM, Allali, Costa J, Muselli A: Qualitative and quantitative analysis of volatile components of Teucrium massiliense $\mathrm{L}$. Identification of 6-methyl-3-heptyl acetate as new natural product. Flavour Fragr J 2010, 25:475-487.

38. Rios JL, Recio MC, Villar A: Antimicrobial activity of selected plants employed in the Spanish Mediterranean area. J Ethnopharmacol 1987, 21:139-152.

39. Cowan MM: Plant products as antimicrobial agents. Clin Microbiol Rev 1999, 12:564-582

40. Lennette FM, Balows A: Manual of Clinical Microbiology. In American Society for Microbiology. Edited by Hansler WJ, Shadomy HJ. Washington, DC; 1985.

doi:10.1186/1752-153X-6-48

Cite this article as: Bendiabdellah et al:: Biological activities and volatile constituents of Daucus muricatus L. from Algeria. Chemistry Central Journal 2012 6:48.

Publish with ChemistryCentral and every
scientist can read your work free of charge
"Open access provides opportunities to our
colleagues in other parts of the globe, by allowing
anyone to view the content free of charge."
W. Jeffery Hurst, The Hershey Company.
- available free of charge to the entire scientific community
- peer reviewed and published immediately upon acceptance
- cited in PubMed and archived on PubMed Central
- yours - you keep the copyright
submit your manuscript here:
http://www.chemistrycentral.com/manuscript/

